nen in Cluster 1 eine deutlich höhere Reaktionsschwelle (Median: $191 \mathrm{mg}$ ) und leichtere Symptome.

Im Cluster 3 „Erdnussallergie mit atopischer Dermatitis und häufiger Kreuzallergie zu Schalen- und/ oder Hülsenfrüchten" fanden sich mit 152 die meisten Kinder wieder. Neben einer atopischen Dermatitis hatten fast $70 \%$ auch ein Asthma. Etwa $40 \%$ litten unter weiteren Nahrungsmittelallergien. Die Reaktionsschwellen und die Stärke der Symptome in dieser Gruppe variierten sehr stark.
Fazit: Die Untersuchung untermauert erneut, wie wichtig es ist, auf die klinische Relevanz zu fokussieren und nicht aufgrund von Sensibilisierungen Verbote auszusprechen. Mithilfe der ermittelten Cluster lassen sich für Therapeuten wichtige Erkenntnisse formulieren:

_-Schwere Erdnussallergien gehen häufig mit allergischen Atemwegsreaktionen einher, zeigen aber trotz vorhandener Sensibilisierung nur bei einem Drittel der Betroffenen Kreuzreaktionen zu Schalen- und Hülsenfrüchten.

\title{
Birkenpollen tagsüber kaum zu entgehen
}

\section{Die lokale Gesamtbelastung der Luft durch Pollen ist von Kräutern und Gräsern größer als durch Bäume. Das lässt eine Studie in Berlin zu Gräser-, Beifuß- und Birkenpollen in der Nähe der Stadtautobahn sowie in der Stadt vermuten.}

In der Studie wurden in den Jahren 2014 und 2015 die Pollenkonzentrationen an zwei verschiedenen Standorten in Berlin im Verlauf der Pollensaison von Gräsern (Poaceae), Beifuß (Artemisia) und Birken (Betula) gemessen. Als Pollensaison wurde der Jahreszeitraum bezeichnet, der $90 \%$ des Pollenindexes, ausgedrückt als Summe der durchschnittlichen täglichen Pollenkonzentrationen, abdeckt. Die sieben Kilometer auseinanderliegenden Standorte der Pollenfallen befanden sich in fünf Meter Höhe an der stark befahrenen Stadtautobahn A100 sowie in 20 Meter Höhe auf dem Dach des AllergieCentrums der Charité. Die Vegetation an der Autobahn war dichter als am innerstädtischen Standort.

Die absoluten Pollenkonzentrationen unterschieden sich deutlich zwischen den beiden Standorten. 2014 wurden an der Autobahn pro Kubikmeter Luft und Tag fast 31.500 Birkenpollen (innerstädtisch: $62 \%$ ), circa 4.300 Gräserpollen (innerstädtisch: 74\%) und mehr als 1.700 Beifußpollen (innerstädtisch: $24 \%$ ) gemessen. Im Jahr darauf hatten sich die Konzentrationen geändert: Die der Birken- und Beifußpollen waren an der Autobahn niedriger, die der Gräserpollen höher als im Vorjahr. In der Stadt waren sowohl die Gräser- als auch die Beifußpollenkonzentrationen gegenüber dem Vorjahr erhöht.
Die Wissenschaftler des Allergie-Centrum-Charité haben darüber hinaus die Pollenbelastung im Verlauf des Tages bestimmt. Demnach wurden die höchsten Konzentrationen an Beifußpollen an beiden Standorten vor allem morgens/spätmorgens gemessen. Bei den Gräserpollen traten die Spitzenwerte an der Autobahn eher gegen Abend auf, innerstädtisch dagegen am Abend und in der Nacht. Schließlich kam es zu Spitzenwerten für die Birkenpollen an der Autobahn nachmittags und nachts, in der Stadt dagegen zwischen 10 und 12 Uhr (2014) sowie zwischen 22 und 24 Uhr (2015). Die vorherrschende Windrichtung am Autobahnstandort zum Zeitpunkt der größten Birkenpollenkonzentration während des Tages war west-östlich, innerstädtisch dagegen kam der Wind zu diesen Zeiten vor allem aus West/Nordwest. Die höchsten Gräserpollenkonzentrationen traten auf, wenn der Wind an beiden Standorten aus West/Nordwest kam.

Aufgrund der Studienergebnisse empfehlen die Wissenschaftler Patienten mit allergischen Reaktionen gegen Beifußpollen, sich vor allem in den frühen und späten Morgenstunden möglichst nicht draußen aufzuhalten und in dieser Zeit nicht die Wohnung zu lüften. Gräserpollenallergiker dagegen hätten möglicherweise morgens weniger Symptome, wenn die Pollenkonzentrationen meist
_Bei hohen Reaktionsschwellen sind klinisch relevante Kreuzallergien unwahrscheinlich.

_Patienten mit atopischer Dermatitis haben ein hohes Risiko, Kreuzallergien zu Schalen- und Hülsenfrüchten, vor allem auf Haselnuss, Cashew und Pistazie zu zeigen. Weitere Nahrungsmittelallergien sind häufig. Dr. Imke Reese

Cousin M et al. Phenotypical characterization of peanut allergic children with differences in cross-allergy to tree nuts and other legumes. Pediatr Allergy Immunol 2017; 28: 245-50

niedrig seien. Dies könne sich aber je nach Gräserart ändern, auch während des Tages und während der Pollensaison. Schließlich fliegen nach Angaben der Forscher Birkenpollen während des ganzen Tages. Spitzenwerte seien zu jeder Zeit des Tages gemessen worden, möglicherweise, weil sie über weite Strecken transportiert werden. Zwar könnten Allergikern keine exakten Empfehlungen gegeben werden, aber zumindest sollten sie in der Pollensaison für Birken zum Beispiel möglichst vor $8 \mathrm{Uhr}$ die Wohnung lüften, nicht später. Peter Leiner

Simoleit A et al. Birch, grass, and mugwort pollen concentrations and intradiurnal patterns at two different urban sites in Berlin, Germany. Allergo J Int 2017; 26: 155-64

\section{Kommentar}

Eine besonders lesenswerte Arbeit für alle, die sich für die Feinheiten des Pollenfluges interessieren. Besonders vor dem Hintergrund, dass Pollenmessstationen in sehr großen Abständen über Deutschland verteilt sind und in der Regel nur Tagesmittelwerte angeben, zeigt diese Arbeit erstmals überzeugend, wie sehr sich das Mikroklima und der Tagesverlauf auf den Pollenflug auswirken kann. Beobachtungen mit direkter Relevanz für die kompetente Beratung unserer Patienten.

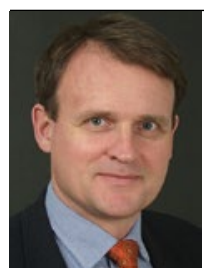

Prof. Dr. Thilo Jakob Direktor der Klinik für Dermatologie und Allergologie Universitätsklinikum Gießen u. Marburg, Standort Gießen 